Med Klin Intensivmed Notfmed $2016 \cdot 111: 4-5$ DOI 10.1007/s00063-015-0128-6

○) Springer-Verlag Berlin Heidelberg 2016

\author{
S. Reith ${ }^{1} \cdot$ H. Hetz ${ }^{2}$ \\ ${ }^{1}$ Medizinische Klinik I, Universitätsklinikum der RWTH Aachen, Aachen, Deutschland \\ ${ }^{2}$ Institut für Anästhesiologie und Intensivmedizin, Unfallkrankenhaus Meidling, Wien, Österreich
}

\title{
Analgesie und Delirmanagement
}

Die intensivmedizinische Behandlung stellt für betroffene Patienten einen dramatischen Einschnitt dar, dessen Folgen die Lebensqualität des Einzelnen auch nach Beendigung des Intensivaufenthalts nachhaltig beeinträchtigen können. Neben der organspezifischen Grunderkrankung leiden kritisch kranke Patienten während der intensivmedizinischen Therapie vor allem an Müdigkeit, Schlaflosigkeit, Störungen des Schlafrhythmus, Durst, Angst, Unruhe, Schmerzen und Verwirrtheitszuständen.

Über viele Jahrzehnte war es daher eine gängige klinische Praxis, intensivpflichtige und beatmete Patienten tief sediert und komplett von der Umwelt abgeschirmt zu behandeln. Die fixe Kombination aus Narkose und kompletter Schmerzausschaltung unter dem Konzept einer tiefen Analgosedierung ist allerdings in den vergangenen Jahren in zahlreichen Publikationen zunehmend kritisch hinterfragt worden. Als wegweisend gilt hier vor allem die Arbeit von Kress et al. [1] aus dem Jahr 2000, in der gezeigt werden konnte, dass ein täglicher Aufwachversuch (,spontaneous awakening trial“) sowohl die Dauer der Beatmung als auch die Dauer des Intensivaufenthalts im Vergleich zu durchgehend tief analgosedierten Patienten signifikant verkürzen konnte. Darüber hinaus konnte zuletzt die Krankheitsentität des Delirs mit seiner variablen und fluktuierenden Beeinträchtigung der Wahrnehmung und des Bewusstseins in seiner Definition näher eingegrenzt werden und entsprechend verstärkt in die differenzialdiagnostischen Überlegungen und den Fokus der Intensivmediziner gelangen.

Als entscheidender Meilenstein des heutigen intensivmedizinischen Standards wurde im Jahr 2005 erstmals für Deutschland die S2k-Leitlinie zur Analgesie und Sedierung in der Intensiv- medizin verfasst. Diese wurde dann im Jahr 2010 unter Federführung der Deutschen Gesellschaft für Anästhesiologie und Intensivmedizin (DGAI) und der Deutschen Interdisziplinären Vereinigung für Intensiv- und Notfallmedizin (DIVI) unter Beteiligung weiterer Fachgesellschaften überarbeitet und als S3-Leitlinie um den Themenkomplex „Delir und Delirmanagement“ erweitert. Im Jahr 2015 liegt nunmehr die nochmals überarbeitete und aktualisierte S3-Leitlinie "Analgesie, Sedierung und Delirmanagement in der Intensivmedizin" vor. Sie integriert die neuesten Erkenntnisse zu diesem Thema mit einer klaren Vorgabe praxisorientierter Handlungsanweisungen. Speziell wird in diesen Leitlinien die eindeutige Empfehlung zu einem wachen aufmerksamen, schmerz-, angst- und delirfreien Patienten auf der Intensivstation ausgesprochen. Hervorgehoben werden der extreme Stellenwert einer adäquaten und dominanten Analgesie sowie die Bedeutung der Prävention und frühzeitigen Diagnosestellung eines Delirs. Gleichzeitig empfehlen die Leitlinien, eine Sedierung innerhalb dieses evidenz- und konsensusorientierten Konzepts nicht mehr regelhaft, sondern nur in speziellen Situationen und bei eindeutig definierten Indikationen durchzuführen.

Der Themenschwerpunkt dieser Ausgabe von Medizinische Klinik - Intensivmedizin und Notfallmedizin soll dem Leser einen prägnanten und praxisorientierten Überblick über den derzeitigen Standard und die aktuellen Empfehlungen zur Analgesie und zum Delirmanagement vermitteln, einer auf jeder Intensivstation bestehenden Problematik und berufsgruppenübergreifenden Herausforderung.

Dabei beleuchten die Beiträge sehr spezifisch die jeweiligen Themen- komplexe des Leitthemas. Der Beitrag von Ortlepp et al. widmet sich dem derzeitigen Therapiestandard der analgetischen Therapie und verdeutlicht dem Leser darüber hinaus sehr klar die Möglichkeiten und Notwendigkeiten der adäquaten Schmerzerfassung und Graduierung.

Eine der häufigsten Komplikationen und größten differenzialdiagnostischen Herausforderungen einer intensivmedizinischen Therapie stellt das Delir dar. Obwohl es sich hierbei prinzipiell um einen vorübergehenden Zustand handelt, ist diese Krankheitsentität mit einer erhöhten Morbidität, Mortalität und langfristigen Pflegebedürftigkeit assoziiert. Der Beitrag von Kersten $u$. Reith beschreibt dieses komplexe klinische Erscheinungsbild und erläutert dabei unter besonderer Berücksichtigung der aktualisierten S3Leitlinien sowohl pharmakologische als auch nichtmedikamentöse Therapieprinzipien.

Gerade auf der internistischen Intensivstation stellen der hämodynamisch kompromittierte Patient mit kardiogenem Schock und der kritisch Kranke mit respiratorischer Insuffizienz und Beatmungspflichtigkeit eine besondere medizinische Herausforderung dar, sowohl bezüglich der organspezifischen als auch hinsichtlich der adjuvanten intensivmedizinischen Therapie.

Der Beitrag von Lemm et al. erläutert die speziellen Aspekte der Analgosedierung und der jeweiligen Substanzauswahl beim kardiogenen Schock. Zur thematischen Komplettierung widmet sich schließlich die Arbeit von Funk der Problematik von Schmerz, Unruhe und Delir beim respiratorisch insuffizienten Patienten. Der Autor beschreibt eindrucksvoll die relevante pathophysiologische und klinische Interaktion zwischen Analgosedierung einerseits und 
Beatmung und Beatmungsform andererseits.

Wir sind zuversichtlich, ein für den Leser informatives und sowohl klinisch als auch wissenschaftlich ansprechendes Leitthema gestaltet zu haben. So verbleiben wir mit dem ausdrücklichen Dank an die Autoren der einzelnen Beiträge.

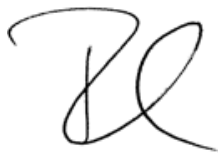

Sebastian Reith

\section{of thibert tuts}

\section{Hubert Hetz}

\section{Korrespondenzadresse}

PD Dr. S. Reith
Medizinische Klinik I,
Universitätsklinikum der
RWTH Aachen
Pauwelsstr. 30, 52074 Aachen
sreith@ukaachen.de

Interessenkonflikt. S. Reith gibt an, dass kein Interessenkonflikt besteht. H. Hetz hat von der Firma Orion Honorare für Fortbildungsveranstaltungen zum Thema Analgosedierung erhalten.

\section{Literatur}

1. Kress JP, Pohlmann AS, O'Connor MF et al (2000) Daily interruption of sedative infusions in critically ill patients undergoing mechanical ventilation. N Engl J Med 342(20):1471-1477

\section{Bundesweites Berichts- \& Lern- system CIRS-AINS bietet Mehr- wert}

Seit April 2010 wurden über 4.000 Berichte in dem bundesweit verbreiteten Critical Incident Reporting System der Anästhesisten, CIRS-AINS, veröffentlicht. Ziel der von dem

Berufsverband der Anästhesisten e. V. (BDA) und der Deutschen Gesellschaft für Anästhesiologie \& Intensivmedizin e. V. (DGAI) betriebenen Datenbank ist eine aktive und nachhaltige Erhöhung der Patientensicherheit durch Sammlung, Auswertung und Weitergabe von Informationen über Ereignisse, Zwischenfälle und Komplikationen mit und ohne Patientenschaden vor, während und nach der Anästhesie. Doch geht das Angebot von CIRS-AINS weit über übliche Standards von medizinischen Incident-Reporting-Systemen hinaus. Bislang nehmen 97 Kliniken an CIRS-AINS teil, davon 13 Universitätskliniken. „Monatlich werden durchschnittlich 50 Fälle gemeldet. Von den jährlich knapp 600 gemeldeten Fällen wurden 345 Ereignisse im Jahr 2014 durch das Expertenteam BDA/DGAI analysiert.

Was aus einem Fallbericht werden kann, zeigt anschaulich der Bericht einer Pflegekraft. Hier gelang es bei einer geplanten Operation nicht, die Narkose und Intubation mit Larynxmaske in Bauchlage des Patienten einzuleiten. Auch auf eine Präoxigenierung wurde verzichtet. Obwohl die Intubation in Bauchlage keine in Lehrbüchern erwähnte und valide Methode ist, ist diese Vorgehensweise gängige Praxis, wie nach der Meldung des Falles festgestellt wurde. So wird das Umlagern umgangen und wertvolle Zeit gespart. Als Ergebnis des Berichtes wurde festgehalten, dass bei der Verwendung der Larynxmaske in Bauchlage vorab Nutzen und Risiken sorgfältig abgewogen werden müssen. Gerade bei Patienten mit pulmonalen Risiken kann der "Goldstandard" Intubation von Vorteil sein. Außerdem erfolgte der dringende Rat, Patienten vor der Narkoseeinleitung grundsätzlich zu präoxygenieren. Dieser Fall wurde nicht nur im Meldesystem CIRS-AINS behandelt. Es folgte eine Umfrage unter den im vertragsärztlichen ambulanten Bereich tätigen Mitgliedern von BDA und DGAI, um Informationen zu Art und Umfang des Einsatzes dieser Narkoseform zu generieren. Die Umfrageergebnisse wurden in der Fachzeitschrift Anästhesiologie \& Intensivmedizin gemeinsam mit der Einschätzung publiziert, dass diese Methode aktuell nicht als sicher empfohlen werden kann. Über das reine Meldesystem „Berichten \& Lernen“ hinaus, wurde so nicht nur der Einzelfall aufgearbeitet, sondern einer Vielzahl von Anästhesisten ein wichtiger Erfahrungswert weitergegeben. Ähnlich verhielt es sich bei Berichten über Partikelkontaminationen nach dem Aufziehen von Arzneimitteln. Die Häufung eingehender Fallberichte führte dazu, dass der Ursache und einer Lösung intensiv nachgegangen wurde. Obwohl schon in der Vergangenheit der Nutzen von Filtersystemen für dieses Problem belegt war, fand deren Verwendung keinen Einzug in die Praxis. Studien hierzu und die Veröffentlichung der Ergebnisse sind nun die Basis für eine Überarbeitung der Fachinformation für Infusionslösungen, die in Planung ist.

Info-Tipp:

Auf der Webseite https://www.cirs-ains.de/ cirs-ains.html haben auch nicht registrierter Nutzer die Möglichkeit, über den Button „Berichten und Lernen" Fallberichte einzugeben und zu lesen. 